\title{
Prevalence and the evaluation of culture, wet mount, and ELISA methods for the diagnosis of Trichomonas vaginalis infection among Ghanaian women using urine and vaginal specimens
}

\author{
Collins Adjei ${ }^{1}$, Richard Boateng ${ }^{2}$, Albert Dompreh², Bismark Okyere ${ }^{1}$ and Eddie-Williams Owiredu ${ }^{3^{*}}$ (D)
}

\begin{abstract}
Background: The services of most clinical laboratories in Africa regarding the diagnosis of Trichomonas vaginalis are largely dependent on the urine direct wet-mount method. However, the exclusive use of urine-based detection may not be appropriate. The culture method is considered the "gold standard" for the diagnosis of T. vaginalis. However, this method has a relatively longer turn-around time and is limited by non-viable organisms in the specimen. This study assessed the prevalence of $T$. vaginalis and its associated risk factors and evaluated its diagnosis using urine and vaginal samples from symptomatic female out-patients by culture, direct wet-mount, and ELISA method respectively.

Methods: This cross-sectional study was conducted at the Obstetrics and Gynaecology department of the Manhyia District hospital (MDH) and Komfo Anokye Teaching Hospital (KATH), Ghana. Ghanaian sexually active female adults between the ages of 18 and 50 years old were recruited for this study. Vaginal (HVS) and urine samples were collected from each participant, and T. vaginalis infection was assessed based on culture, direct wet mount, and ELISA methods.

Results: The prevalence of T. vaginalis infection based on the ELISA method, HVS culture, and HVS wet mount were $7.2 \%, 5.0 \%$, and $1.7 \%$, respectively. Urine culture presented with a $0.6 \%$ prevalence rate while urine direct wet mount detected no positive case. There was no statistically significant association between demographic and clinical characteristics and $T$. vaginalis infection, except for subjects presenting with abdominal pain $[O R=5.42,95 \%$ $\mathrm{Cl}(1.35-21.73), p=0.017]$. Using HVS culture as the reference, ELISA performed best compared to the other methods assessed in this study, presenting with the highest sensitivity [88.9\%, 95\% Cl (54.0-99.8)], specificity [97.1\%, 95\% Cl (93.1-98.9)], AUC (93.0\%), and accuracy (96.7\%).

Conclusion: The prevalence of $T$. vaginalis infection is high among women in Ghana. With the exception of abdominal pain, there is no significant association between demographic and clinical characteristics and T. vaginalis infection. In the event where the culture method is unavailable or when rapid diagnosis is required, antigenic detection using ELISA is the most accurate for the diagnosis of $T$. vaginalis infection in women compared to urine wet-mount/culture and the HVS wet-mount method.
\end{abstract}

Keywords: Trichomonas vaginalis, Culture, Wet mount, ELISA

\footnotetext{
* Correspondence: eddiewilliams.owiredu@gmail.com

${ }^{3}$ Department of Molecular Medicine, School of Medical Sciences, College of

Health Sciences, Kwame Nkrumah University of Science and Technology,

Kumasi, Ghana

Full list of author information is available at the end of the article
}

(c) The Author(s). 2019 Open Access This article is distributed under the terms of the Creative Commons Attribution 4.0 International License (http://creativecommons.org/licenses/by/4.0/), which permits unrestricted use, distribution, and

reproduction in any medium, provided you give appropriate credit to the original author(s) and the source, provide a link to the Creative Commons license, and indicate if changes were made. The Creative Commons Public Domain Dedication waiver (http://creativecommons.org/publicdomain/zero/1.0/) applies to the data made available in this article, unless otherwise stated. 


\section{Background}

Trichomoniasis, an infectious disease which mostly occurs in women of reproductive age, is increasing globally. It accounted for 59.7 million new cases of infection in 2008, with 42.8 million infections at any point in time [1]. Currently, the WHO reports 143 million new infections annually [2]. In Africa, a prevalence of $25.64 \%$ has been reported [3].

Trichomonas vaginalis, a parasitic protozoan, is the causative agent for trichomoniasis. It infects the urogenital tract and has been associated with urethritis, vaginitis, cervicitis, pelvic inflammatory disease, and tubal infertility [4-7]. Recently, T. vaginalis has been shown to facilitate the acquisition of HIV [8] as well as cancer [9, 10], preterm delivery, and low birth weight [11].

Due to the several detrimental effects associated with the infection, accuracy and precision in clinical diagnosis is essential to reduce the risk of transmission and morbidity. Direct wet-mount microscopic examination is the most used method for $T$. vaginalis diagnosis, especially in low-and middle-income countries such as Ghana because it is simple and inexpensive [12, 13]. However, direct wet-mount microscopy has low sensitivity and is subject to diagnostic biases as a result of its dependence on operator's experience and viability of the organism in the specimen $[14,15]$. The introduction of staining to the direct microscopic method attempted to solve these problems, but reports indicate no increase in sensitivity compared with the unstained direct wet mount method [16].

Cultivation of $T$. vaginalis is more sensitive and valuable especially when the amount of inoculum is few [17]. As such, the culture technique is considered the "gold standard" for the diagnosis of T. vaginalis [18]. Nonetheless, this method is limited in the event of non-viable organisms in specimen [16]. Furthermore, in addition to the relatively longer turn-around time of 2-7 days, not many clinical laboratories in Africa are equipped to accurately perform the culture-based detection method. Recently, the sensitivity of $T$. vaginalis diagnosis has been greatly improved by the use of nucleic acid amplification techniques [15]. However, this technique is expensive and not readily available in many resource-limited settings. As a result, serological methods using monoclonal antibodies have been advocated for detection of $T$. vaginalis. These include latex agglutination, immunofluorescence, enzyme-linked immunosorbent assay (ELISA), and lateral flow techniques [16, 19]. Nevertheless, these serological techniques may be technically demanding. As such, each technique has its own merits and demerits. In Ghana, however, services of most clinical laboratories regarding the diagnosis of $T$. vaginalis are limited to the direct wet-mount microscopic technique using urine as the specimen. Nonetheless, report indicates that exclusive use of urine-based detection of $T$. vaginalis may not be appropriate [14].
This study assessed the prevalence of $T$. vaginalis and its associated risk factors and evaluated its diagnosis using urine and vaginal samples from female out-patients by the direct wet-mount, culture, and ELISA technique, respectively. The finding of this study would equip health service providers with limited resources, the information on which techniques may work best to ensure informed and accurate clinical decisions in this era of evidence-based medicine.

\section{Materials and methods Study design/area}

This cross-sectional study was conducted between December, 2016, and September, 2017, at the Obstetrics and Gynaecology department of the Manhyia District hospital (MDH) and Komfo Anokye Teaching Hospital (KATH), both in Kumasi, Ghana. Kumasi has a projected population of 4,780,380 individuals, accounting for $19.4 \%$ of Ghana's total population. The entire Manhyia district is served by $\mathrm{MDH}$, and $\mathrm{KATH}$ is the second largest hospital in Ghana, serving patients from the upper part of the country [20].

\section{Study population}

The sample size for this study was calculated using the Raosoft sample size calculator [21]. At 95\% confidence level, $7 \%$ margin of error, and a response distribution of $50 \%$, a total of 180 symptomatic Ghanaian sexually active female adults aged $18-50$ years were recruited for the study.

\section{Inclusion and exclusion criteria}

This study included only symptomatic female patients. Patients attending the out-patient clinic were approached, and the study objectives were explained to them. Subjects who agree to participate in the study provided written informed consent. Included participants were non-pregnant, were not menstruating, and have not had sexual intercourse at least 3 days prior to sampling. Patients who were unwilling to participate and those who had been on antiprotozoal, antibiotics, or steroids for the past 2 weeks were excluded from the study.

\section{Questionnaire administration and clinical data extraction}

A validated questionnaire, designed by reviewing previous studies of similar objectives and adjusted to suit our study objective, was used to obtain relevant socio-demographic and clinical data from each respondent.

\section{Sample collection and laboratory analysis}

Two (2) consecutive vaginal swab samples were collected from the posterior fornix of the vagina using sterile cotton wool swab sticks by specialised female laboratory scientists. The first sample was transferred 
into $1 \mathrm{ml}$ of $0.85 \%$ sterile normal saline and mixed thoroughly. A drop of the mixture was placed on a clean, grease-free microscope slide, covered with a coverslip and observed under light microscope using $\times 10$ objective lens for motile trichomonads, followed by confirmation with $\times 40$ objective lens. In order to increase the chances of recovering the $T$. vaginalis, three slides were prepared for each specimen. Due to the delicate nature of the organism outside its normal vaginal environment, all microscopic examinations were performed within 30 min of sample collection. Specimens with the presence of $T$. vaginalis trophozoites (one or more motile trichomonads) were considered positive by vaginal sample (HVS) wet-mount method. The remainder of the first sample was stored at $-20^{\circ} \mathrm{C}$ until $T$. vaginalis antigen testing by ELISA method. The second vaginal swab was inoculated into Kupferberg culture medium (HIMEDIA Laboratories, Mumbai, India) after collection and incubated at $37^{\circ} \mathrm{C}$, with daily microscopic examination for the presence of $T$. vaginalis trophozoites. Negative specimens were continually incubated in the same condition until the 7th day after the initial inoculation, after which samples with no trophozoites upon microscopic examination were considered negative for $T$. vaginalis by HVS culture method.

T. vaginalis antigen detection was performed by sandwich ELISA method (Kalon Biological, Guildford, UK) according to the manufacturer's instructions. Briefly, $50 \mu \mathrm{L}$ of positive control, negative control, and patient sample were pipetted into respective microtitre wells on a microtitre plate. Enzyme conjugate reagent $(100 \mu \mathrm{L})$ was added. The content of each well was mixed thoroughly, covered with an adhesive cover, and incubated at $37^{\circ} \mathrm{C}$ for $60 \mathrm{~min}$. After incubation, the mixture was aspirated from the wells followed by four washes with the wash solution. Residual wash solution droplets were removed by blotting the microtitre plate onto an absorbent paper. A $100 \mu \mathrm{L}$ of tetramethylbenzidine (TMB) solution (prepared from $50 \mu \mathrm{L}$ of chromogen $\mathrm{A}$ and $50 \mu \mathrm{L}$ of chromogen B) was pipetted into each well, mixed gently, and incubated at $37^{\circ} \mathrm{C}$ for $15 \mathrm{~min}$. Fifty (50) $\mu \mathrm{L}$ of Stop Solution was added to each well and gently mixed for $30 \mathrm{~s}$ to stop the reaction. The absorbance of each well was measured spectrophotometrically at $450 \mathrm{~nm}$ using Thermo Electron Multiskan EX plate reader (Shanghai, China).

After collection of vaginal samples, each respondent was asked to provide about $20-40 \mathrm{ml}$ of urine sample. Urine was collected into clean, grease-free, wide-mouth urine containers. The urine was mixed thoroughly; aliquots of $10 \mathrm{ml}$ was prepared and centrifuged at 1000 $\mathrm{rpm}$ for $5 \mathrm{~min}$. The supernatant was decanted, and a drop of the sediment was placed on a clean, grease-free microscope slide, covered with a cover slip, and examined following the same protocol used for the vaginal swab specimen. The remaining urine sediment was re-suspended in distilled water, and $100 \mu \mathrm{l}$ of the suspension was pipetted into Kupferberg culture medium, followed by incubation at $37^{\circ} \mathrm{C}$ for 7 days. The protocol for incubation and examination of vaginal swab specimen was similarly applied to the urine specimen. All laboratory analyses were conducted under standard laboratory conditions.

\section{Statistical analysis}

Data processing was done using Microsoft Excel 2016. Statistical analysis and graphical presentation was performed using the R Language for Statistical Computing version 3.5.2 (R Core Team, Vienna, Austria) [22]. Categorical data were presented as frequency (percentages). Univariate logistic regression analysis was used to assess the association between sociodemographic and clinical characteristics and $T$. vaginalis infection. The kappa (к) statistic was used to evaluate the agreement between the various tests used in the study and the receiver operating characteristics (ROC) curve analysis was used to assess the diagnostic performance of the tests. Confidence was set at $95 \%$, and a $p$ value $<0.05$ was considered statistically significant.

\section{Results}

Table 1 shows the demographic and clinical characteristics of the study population. A higher proportion of the subjects were $21-30$ years old $(52.2 \%)$, were married (65.6\%), had basic education (49.4\%), had a single sexual partner (85.0\%), were unemployed (70.6\%), and were living in the urban setting (92.8\%). Additionally, a higher proportion of the vaginal samples were whitish in colour (44.4\%). Furthermore, the prevalence of vaginal odour, vaginal itchiness, lower abdominal pain, and dysuria were $21.7 \%, 36.7 \%, 14.4 \%$, and $7.2 \%$, respectively (Table 1).

The prevalence of $T$. vaginalis infection stratified by type of specimen and test methods applied is shown in Table 2. The prevalence of $T$. vaginalis infection based on the ELISA method, HVS culture, and HVS wet mount were $7.2 \%, 5.0 \%$, and $1.7 \%$, respectively. Urine culture presented with a $0.6 \%$ prevalence rate. However, urine wet mount did not detect any parasite (Table 2).

Table 3 shows the association between demographic and clinical characteristics and T. vaginalis infection. There was no statistically significant association between demographic and clinical characteristics and $T$. vaginalis infection, except women presenting with abdominal pain where an increased odds of $T$. vaginalis infection was observed [OR $=5.42,95 \%$ CI $(1.35-21.73), p=0.017]$ compared to participants who presented with no abdominal pain (Table 3). 
Table 1 Demographic and clinical characteristics of the study population

\begin{tabular}{|c|c|c|}
\hline Variables & Frequency $(n=180)$ & Percentage \\
\hline \multicolumn{3}{|c|}{ Demographic characteristics } \\
\hline \multicolumn{3}{|l|}{ Age (years) } \\
\hline$<20$ & 25 & 13.9 \\
\hline $21-30$ & 94 & 52.2 \\
\hline $31-40$ & 50 & 27.8 \\
\hline $41-50$ & 11 & 6.1 \\
\hline \multicolumn{3}{|l|}{ Marital status } \\
\hline Single & 62 & 34.4 \\
\hline Married & 118 & 65.6 \\
\hline \multicolumn{3}{|l|}{ Educational level } \\
\hline No formal education & 9 & 5.0 \\
\hline Basic & 89 & 49.4 \\
\hline Secondary & 50 & 27.8 \\
\hline Tertiary & 32 & 17.8 \\
\hline \multicolumn{3}{|l|}{ Residence } \\
\hline Rural & 13 & 7.2 \\
\hline Urban & 167 & 92.8 \\
\hline \multicolumn{3}{|l|}{ Employment status } \\
\hline Employed & 53 & 29.4 \\
\hline Unemployed & 127 & 70.6 \\
\hline \multicolumn{3}{|c|}{ Number of sexual partners } \\
\hline 0 & 4 & 2.2 \\
\hline 1 & 153 & 85.0 \\
\hline 2 & 19 & 10.6 \\
\hline 3 & 4 & 2.2 \\
\hline \multicolumn{3}{|l|}{ Clinical characteristics } \\
\hline \multicolumn{3}{|c|}{ Colour of vaginal sample } \\
\hline Clear & 58 & 32.2 \\
\hline Whitish & 80 & 44.4 \\
\hline Yellowish-green & 42 & 23.3 \\
\hline Vaginal odour & 39 & 21.7 \\
\hline Itchiness & 66 & 36.7 \\
\hline Abdominal pain & 26 & 14.4 \\
\hline Dysuria & 13 & 7.2 \\
\hline
\end{tabular}

Table 2 The prevalence of $T$. vaginalis infection stratified by type of specimen and test methods applied

\begin{tabular}{lll}
\hline Test method & Positive & Negative \\
\hline ELISA & $13(7.2)$ & $167(92.8)$ \\
Vaginal sample (HVS) culture & $9(5.0)$ & $171(95.0)$ \\
Vaginal sample (HVS) wet mount & $3(1.7)$ & $177(98.3)$ \\
Urine culture & $1(0.6)$ & $179(99.4)$ \\
Urine wet mount & $0(0.0)$ & $180(100.0)$ \\
\hline
\end{tabular}

ELISA enzyme-linked immunosorbent assay
Table 3 Association between demographic and clinical characteristics and T. vaginalis infection

\begin{tabular}{|c|c|c|c|c|}
\hline Variables & Negative & Positive & OR $(95 \% \mathrm{Cl})$ & $p$ value \\
\hline \multicolumn{5}{|c|}{ Demographic characteristics } \\
\hline \multicolumn{5}{|l|}{ Age (years) } \\
\hline$<20$ & $25(100)$ & $0(0.0)$ & 1 & \\
\hline $21-30$ & $88(93.6)$ & $6(6.4)$ & $3.75(0.20-68.76)$ & 0.374 \\
\hline $31-40$ & $47(94.0)$ & $3(6.0)$ & $3.76(0.19-75.63)$ & 0.387 \\
\hline $41-50$ & $11(100.0)$ & $0(0.0)$ & - & \\
\hline \multicolumn{5}{|l|}{ Marital status } \\
\hline Single & $59(95.2)$ & $3(4.8)$ & 1 & \\
\hline Married & $112(94.9)$ & $6(5.1)$ & $1.05(0.25-4.36)$ & 0.943 \\
\hline \multicolumn{5}{|l|}{ Educational level } \\
\hline No formal education & $8(88.9)$ & $1(11.1)$ & 1 & \\
\hline Basic & $87(97.8)$ & $2(2.2)$ & $0.18(0.01-2.26)$ & 0.186 \\
\hline Secondary & $48(96.0)$ & $2(4.0)$ & $0.33(0.03-4.12)$ & 0.392 \\
\hline Tertiary & $28(87.5)$ & $4(12.5)$ & $1.14(0.11-11.72)$ & 0.911 \\
\hline \multicolumn{5}{|l|}{ Residence } \\
\hline Rural & $13(100.0)$ & $0(0.0)$ & 1 & \\
\hline Urban & $158(94.6)$ & $9(5.4)$ & $1.62(0.09-29.34)$ & 0.745 \\
\hline \multicolumn{5}{|l|}{ Employment status } \\
\hline Employed & $52(98.1)$ & $1(1.9)$ & 1 & \\
\hline Unemployed & $119(93.7)$ & $8(6.3)$ & $3.50(0.43-28.67)$ & 0.244 \\
\hline
\end{tabular}

Number of sexual partners

$\begin{array}{lllll}0 & 4(100.0) & 0(0.0) & 1 & \\ 1 & 145(94.8) & 8(5.2) & 0.53(0.03-10.57) & 0.675 \\ 2 & 18(94.7) & 1(5.3) & 0.73(0.03-21.06) & 0.854 \\ 3 & 4(100.0) & 0(0.0) & 1.00(0.02-62.31) & 1.000\end{array}$

Clinical characteristics

Odour

$\begin{array}{lllll}\text { No } & 134(95.0) & 7(5.0) & 1 & \\ \text { Yes } & 37(94.9) & 2(5.1) & 1.03(0.21-5.19) & 0.967\end{array}$

Colour of vaginal sample

$\begin{array}{lllll}\text { Clear } & 55(94.8) & 3(5.2) & 1 & \\ \text { Whitish } & 78(97.5) & 2(2.5) & 0.47(0.08-2.91) & 0.417 \\ \text { Yellowish-green } & 38(90.5) & 4(9.5) & 1.93(0.41-9.12) & 0.407 \\ \text { Dysuria } & & & & \\ \text { No } & 159(95.2) & 8(4.8) & 1 & 0.647 \\ \text { Yes } & 12(92.3) & 1(7.7) & 1.66(0.19-14.36) & \end{array}$

$\begin{array}{lllll}\text { No } & 108(94.7) & 6(5.3) & 1 & \\ \text { Yes } & 63(95.5) & 3(4.5) & 0.86(0.21-3.55) & 0.832 \\ \text { Abdominal pain } & & & & \\ \text { No } & 149(96.8) & 5(3.2) & 1 & \\ \text { Yes } & 22(84.6) & 4(15.4) & 5.42(1.35-21.73) & 0.017\end{array}$

Univariate logistic regression analysis was used to assess the association between demographic and clinical characteristics and $T$. vaginalis infection based on prevalence by the gold standard (HVS culture). $p$ values $<0.05$ were considered statistically significant ( $p$ values of significant variables in italics)

$O R$ odds ratio 
As shown in Fig. 1, of the nine (9) cases diagnosed as positive for T. vaginalis using the gold standard (HVS culture), eight (8) were identified as positive by the ELISA method with a substantial agreement $(\kappa=0.710)$ (Fig. 1a), three (3) by the HVS wet mount method with a moderate agreement $(\kappa=0.487)$ (Fig. 1b), and one (1) by the urine culture method with a slight agreement $(\kappa=0.192)$ (Fig. 1c). None of the urine samples were positive for $T$. vaginalis using the wet mount method (Fig. 1d).

We used the receiver operating characteristics (ROC) curve analysis to evaluate the performance of the test methods assessed in this study using HVS culture as the reference, as shown in Fig. 2 and Table 4. ELISA performed best compared to the other methods used in this study, presenting with the highest sensitivity [88.9\%, 95\% CI (54.0-99.8)], specificity [97.1\%, 95\% CI (93.1-98.9)], AUC (93.0\%), and accuracy (96.7\%). With an accuracy of $96.7 \%$, HVS wet mount presented with a specificity of [100\%, 95\% CI (97.3-100.0)] but a low sensitivity [33.3\%, 95\% CI (12.0-64.9)] and AUC (66.7\%). Additionally, despite the high specificity [100\%, 95\% CI (97.2-100.0)] for urine culture, there was a lower sensitivity [11.1\%, 95\% CI (0.2-45.9)] and accuracy (55.6\%) (Table 4).

\section{Discussion}

The prevalence of $T$. vaginalis infection in this present study using the "gold standard" (HVS culture) was 5.0\%. This finding is in harmony with the finding of a cross-sectional study by Mahmoud et al. among Egyptian women [16]. In their study, fifty (50) out of 1000 symptomatic female patients were positive for $T$. vaginalis infection. The finding of this present study is also consistent with a cross-sectional study by Al-Saeed et al. who reported a prevalence of $5.4 \%$ among a total of 425 vaginal swabs collected from women from Dohok province in Iraq using the culture-based detection method [23]. However, a recent study by Asmah et al. in Ghana reported that 64 of 150 outpatients were positive for $T$. vaginalis [12]. This discrepancy may be due to the fact that Asmah et al. included both males and females in
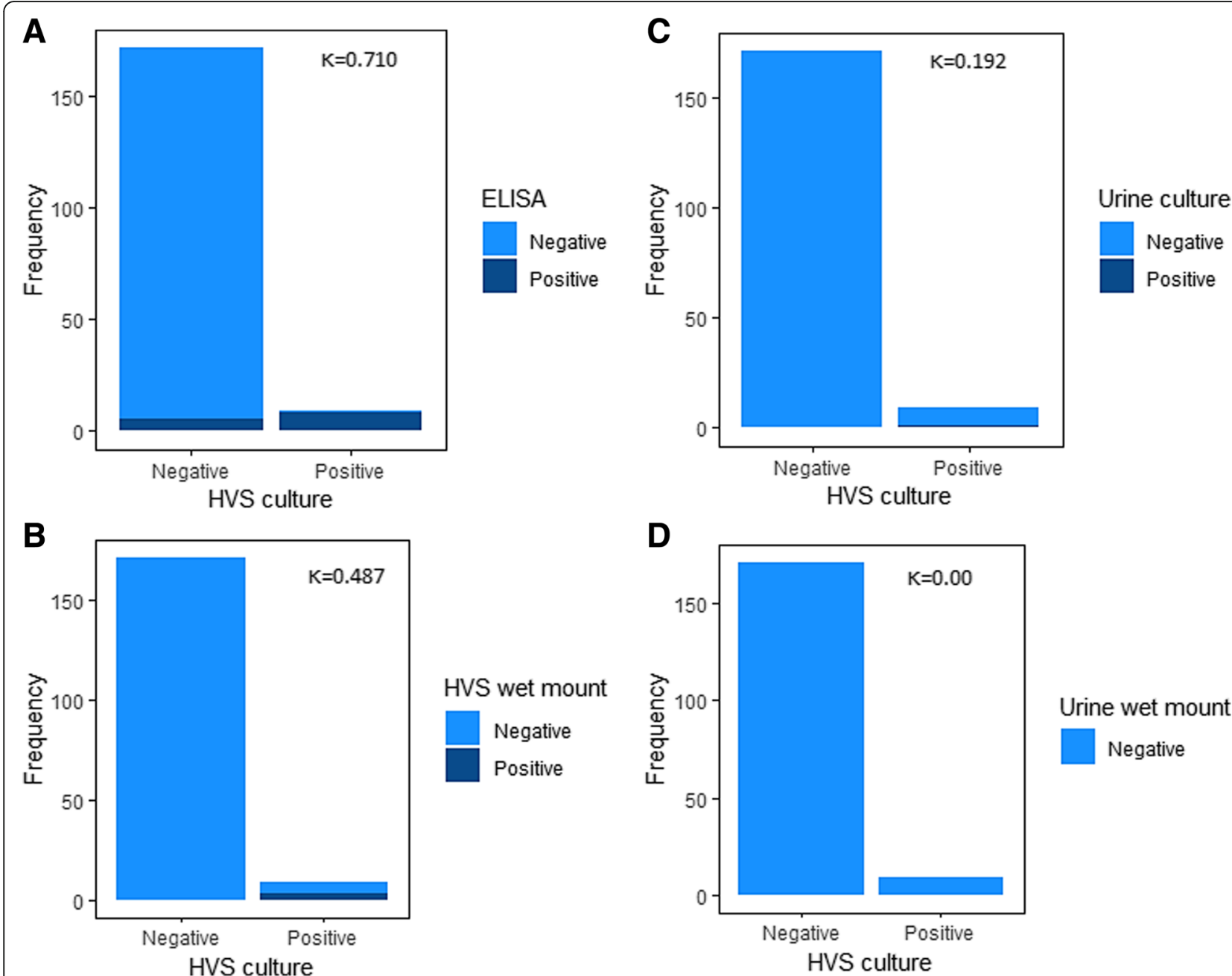

Urine wet mount


Fig. 1 Concordance evaluation between the use of HVS culture and the other tests 


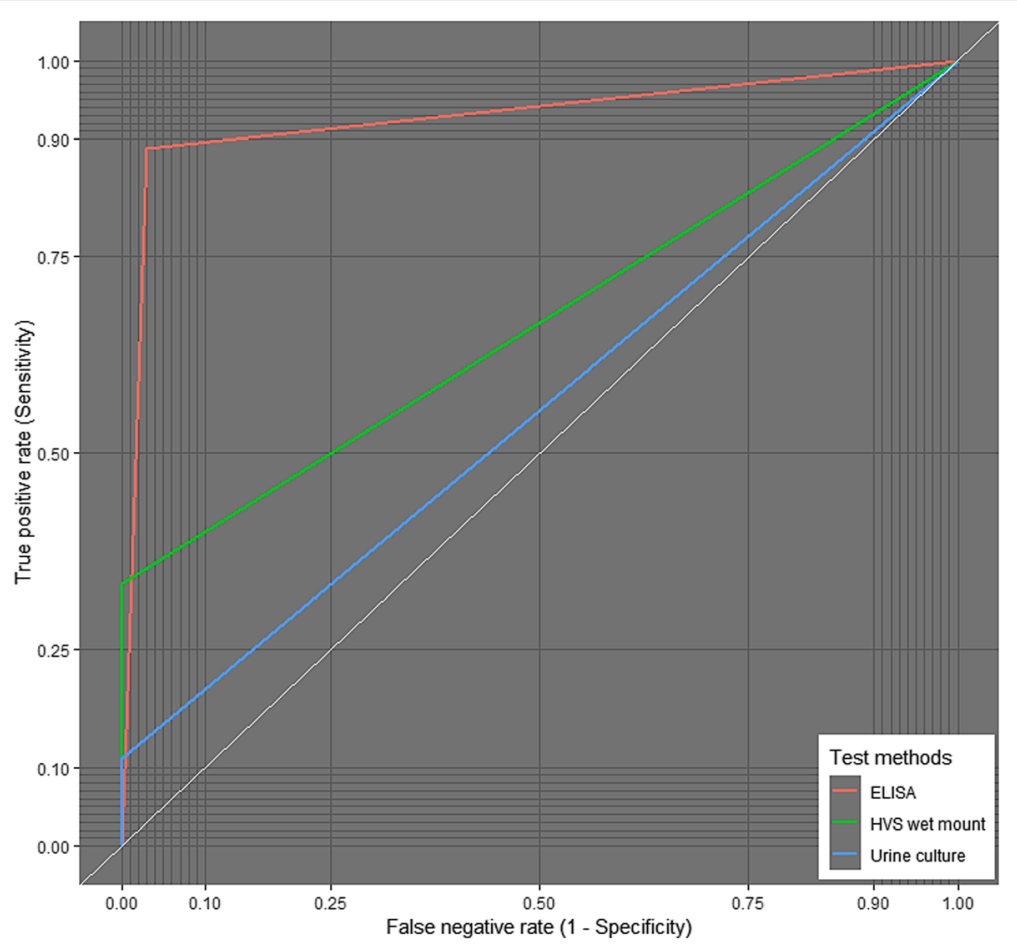

Fig. 2 Evaluation of the performance of test methods used

their study while only females were included in this present study. Additionally, they employed polymerase chain reaction (PCR) technique while we did not, which may have underestimated the prevalence rate obtained in this study. Moreover, their study was originally conducted in 2014, and increasing level of knowledge and awareness of the infection may have resulted in the diminution of the prevalence rate. Furthermore, geographical dissimilarities may be partly involved in the differences in prevalence rates because their study was

Table 4 Evaluation of the performance of test methods used

\begin{tabular}{llll}
\hline Methods* $^{*}$ & ELISA & HVS wet mount & Urine culture \\
\hline Sensitivity (95\% Cl) & $88.9(54.0-99.8)$ & $33.3(12.0-64.9)$ & $11.1(0.2-45.9)$ \\
Specificity (95\% Cl) & $97.1(93.1-98.9)$ & $100(97.3-100.0)$ & $100(97.2-100.0)$ \\
PPV & 61.5 & 100.0 & 100.0 \\
NPV & 99.4 & 96.7 & 95.3 \\
TP & 8 & 3 & 1 \\
TN & 166 & 171 & 163 \\
FP & 5 & 0 & 0 \\
FN & 1 & 6 & 8 \\
Accuracy (\%) & 96.7 & 96.7 & 95.4 \\
AUC (\%) & 93.0 & 66.7 & 55.6 \\
\hline
\end{tabular}

$P P V$ positive predictive value, $N P V$ negative predictive value, $T P$ true positives, $T N$ true negatives, $F P$ false positives, $F N$ false negatives, $A U C$ area under curve *Urine wet mount has been excluded due to lack of positivity conducted in southern Ghana while this current study took place in northern Ghana.

Another finding of this study is that there was no significant association between demographic and clinical characteristics with $T$. vaginalis infection, except for subjects presenting with abdominal pain, where an increased odds of $T$. vaginalis infection was observed compared with participants who presented with no abdominal pain. Coherent with this finding is a cross-sectional study by Madhivanan et al. among 200 non-pregnant female patients in Egypt [24]. Madhivanan et al. reported no significant association between potential risk factors and $T$. vaginalis infection based on vaginal sample culture using InPouch TV culture kit, with the exception of patients complaining of either dysuria, dyspareunia, or abdominal pain. This finding is also consistent, in part, with a study by Fernando et al. in Sri Lanka [25] among female clinic attendees aged 15-60 years. They reported no significant association between demographic and clinical characteristics and T. vaginalis infection based on vaginal sample culture. However, the association between demographic and clinical characteristics with $T$. vaginalis infection remains debatable as there have been reports of significant association with age, ethnicity, and education [26-28]. Verteramo et al. [26] and Helms et al. [27] found a significant associations between $T$. vaginalis infection with older age and low level of education among women in Italy and USA 
respectively using the modified Diamond's medium. Another study by Sutton et al. [28] among reproductive-age women in the USA reported that ethnicity, being born in the USA, increasing age, and lower educational level were associated with increased risk of $T$. vaginalis infection based on PCR. Nonetheless, it is worthy of note that, these studies employed more sensitive methods for the detection of $T$. vaginalis. A more sensitive method results in the identification of higher number of positive cases, which may have been missed in this present study, consequently influencing risk associations.

The symptoms of $T$. vaginalis infection are non-specific. As such, diagnosis largely depends on the use of clinical laboratory techniques. Currently, the most commonly used laboratory technique for $T$. vaginalis diagnosis, especially in Africa, is the wet mount method because it is simple and inexpensive [13]. In this present study, direct wet mount using urine specimen did not detect any positive case of $T$. vaginalis. Urine culture, on the other hand, resulted in a $0.6 \%$ prevalence rate with a specificity of $100 \%$, sensitivity of $11.1 \%$, and accuracy of $55.6 \%$ whereas direct wet mount examination of vaginal specimen resulted in a prevalence of $1.7 \%$ [specificity $=$ $100 \%$, sensitivity $=33.3 \%$, and $\mathrm{AUC}=66.7 \%$, suggesting that the exclusive use of urine-based detection of $T$. vaginalis may not be appropriate. This finding is in unison with a study by Lawing et al. in USA [14] and Patil et al. in India [29].

The prevalence of $T$. vaginalis infection was highest with ELISA-based antigenic detection method (7.2\%). Using the "gold standard" as the reference, the use of ELISA presented with a substantial concordance with the vaginal sample culture method $(\kappa=0.710)$, with vaginal sample wet mount and urine culture methods presenting with moderate $(\kappa=0.487)$ and slight agreement $(\kappa=0.192)$ respectively. In order to evaluate the performance of each test method in diagnosing $T$. vaginalis infection, we employed the receiver operating characteristics (ROC) curve analysis with reference to the gold standard (vaginal sample culture). The ELISA method performed best compared to the other methods, presenting with the highest sensitivity [88.9\%, 95\% CI $(54.0-$ 99.8)], specificity [97.1\%, 95\% CI (93.1-98.9)], AUC (93.0\%), and accuracy (96.7\%). This suggest that antigenic detection using ELISA-based method may be used as a surrogate to HVS culture, for accurate diagnosis of $T$. vaginalis infection in women in the event where the culture method is unavailable or when rapid diagnosis is required. Nonetheless, it is worthy of note that there is a false-negative and false-positive rate of $1.1 \%$ and $2.9 \%$ respectively when the ELISA method was used compared with the vaginal sample culture method; hence, results from the ELISA method should be interpreted with caution. This incongruity may be partly associated with the detection of nonpathogenic trichomonads such as Pentatrichomonas hominis [30], probably due to crosscontamination between the anorectal and cervico-vaginal sites, by the ELISA method possibly due to cross-reactivity [31, 32]. Alternatively, the disparity may be linked to the possibility of non-viable $T$. vaginalis in the culture specimen. This is because the culture-based method is chiefly dependent on the viability of the organisms and the presence of non-viable organism will result in a negative culture result [16].

This study is however limited by the fact that we did not perform the more sensitive polymerase chain reaction (PCR) method and this may have underestimated the overall prevalence of the infection.

\section{Conclusions}

The prevalence of $T$. vaginalis infection is high among women in Ghana. With the exception of abdominal pain, there is no significant association between demographic and clinical characteristics and $T$. vaginalis infection. We, thus, recommend increasing the health awareness of females to undertake regular check-ups, especially when they experience abdominal pains. In the event where the culture method is unavailable or when rapid diagnosis is required, antigenic detection using ELISA is the most accurate for the diagnosis of $T$. vaginalis infection in women compared to urine wet-mount/culture and the HVS wet-mount method.

\section{Abbreviations}

ELISA: Enzyme-linked immunosorbent assay; HVS: High vaginal swab; KATH: Komfo Anokye Teaching Hospital; MDH: Manhyia District hospital

\section{Acknowledgements}

The authors are grateful to the Staff of the Manhyia District hospital and the Komfo Anokye Teaching Hospital and all who actively participated in the study.

\section{Funding}

This research did not receive any specific grant from funding agencies in the public, commercial, or not-for-profit sectors.

\section{Availability of data and materials}

The datasets used and/or analysed during the current study are available from the corresponding author on reasonable request.

\section{Authors' contributions \\ $C A, R B, A D$, and $B O$ were involved in the design of the study, collection of data, laboratory analysis, and drafting and revision of the manuscript. EWO was involved in the design of the study, collection of data, laboratory analysis, statistical analysis and interpretation, and drafting and revision of the manuscript. All authors read and approved the final manuscript.}

\section{Ethics approval and consent to participate}

Ethical approval for this study was obtained from the committee on Human Research, Publications and Ethics (CHRPE) of the School of Medical Sciences of the Kwame Nkrumah University of Science and Technology (CHRPE/AP/ 497/16) and also from the research and development department of KATH and $\mathrm{MDH}$. Written informed consent was obtained from all participants who opted to participate after the aims and objectives of the study had been explained to them. Participation was voluntary, and respondents were assured that the information obtained was strictly for research and academic 
purposes only and were guaranteed the liberty to opt out from the study at their own convenience.

\section{Consent for publication \\ Not applicable.}

\section{Competing interests}

The authors declare that they have no competing interests.

\section{Publisher's Note}

Springer Nature remains neutral with regard to jurisdictional claims in published maps and institutional affiliations.

\section{Author details}

'Department of Clinical Microbiology, School of Medical Sciences, College of Health Sciences, Kwame Nkrumah University of Science and Technology, Kumasi, Ghana. ${ }^{2}$ Department of Clinical Microbiology, Komfo Anokye Teaching Hospital, Kumasi, Ghana. ${ }^{3}$ Department of Molecular Medicine, School of Medical Sciences, College of Health Sciences, Kwame Nkrumah University of Science and Technology, Kumasi, Ghana.

Received: 25 March 2019 Accepted: 3 May 2019

Published online: 16 May 2019

\section{References}

1. WHO. Global incidence and prevalence of selected curable sexually transmitted infections - 2008. 2012.

2. Sexually transmitted infections (STIs): WHO; 2019 .

3. WHO. Prevalence and incidence of selected sexually transmitted infections, Chlamydia trachomatis, Neisseria gonorrhoeae, Syphilis and trichomonas vaginalis: methods and results used by WHO to generate 2005 estimates. 2011.

4. Lee JJ, Moon HS, Lee TY, Hwang HS, Ahn M-H, Ryu J-S. PCR for diagnosis of male trichomonas vaginalis infection with chronic prostatitis and urethritis. Korean J Parasitol. 2012;50(2):157.

5. Campbell L, Woods V, Lloyd T, Elsayed S, Church D. Evaluation of the OSOM trichomonas rapid test versus wet preparation examination for detection of trichomonas vaginalis vaginitis in specimens from women with a low prevalence of infection. J Clin Microbiol. 2008;46(10):3467-9.

6. Hobbs MM, Seña AC. Modern diagnosis of trichomonas vaginalis infection Sex Transm Infect. 2013;89(6):434-8

7. Moodley P, Wilkinson D, Connolly C, Moodley J, Sturm AW. Trichomonas vaginalis is associated with pelvic inflammatory disease in women infected with human immunodeficiency virus. Clin Infect Dis. 2002;34(4):519-22.

8. McClelland RS, Sangaré L, Hassan WM, Lavreys L, Mandaliya K, Kiarie J, et al. Infection with trichomonas vaginalis increases the risk of HIV-1 acquisition. J Infect Dis. 2007;195(5):698-702.

9. Stark JR, Judson G, Alderete JF, Mundodi V, Kucknoor AS, Giovannucci EL, et al. Prospective study of trichomonas vaginalis infection and prostate cancer incidence and mortality: Physicians' health study. J Natl Cancer Inst. 2009; 101(20):1406-11.

10. Fowke JH, Han X, Alderete J, Moses KA, Signorello LB, Blot WJ. A prospective study of trichomonas vaginalis and prostate cancer risk among African American men. BMC research notes. 2016;9(1):224.

11. Silver BJ, Guy RJ, Kaldor JM, Jamil MS, Rumbold AR. Trichomonas vaginalis as a cause of perinatal morbidity: a systematic review and meta-analysis. Sex Transm Dis. 2014;41(6):369-76.

12. Asmah RH, Agyeman RO, Obeng-Nkrumah N, Blankson $H$, Awuah-Mensah $\mathrm{G}$, Cham M, et al. Trichomonas vaginalis infection and the diagnostic significance of detection tests among Ghanaian outpatients. BMC Womens Health. 2018;18(1):206.

13. Bachmann LH, Hobbs MM, Sena AC, Sobel JD, Schwebke JR, Krieger JN, et al. Trichomonas vaginalis genital infections: progress and challenges. Clin Infect Dis. 2011;53(suppl_3):S160-S72.

14. Lawing LF, Hedges SR, Schwebke JR. Detection of trichomonosis in vaginal and urine specimens from women by culture and PCR. J Clin Microbiol. 2000;38(10):3585-8.

15. Wendel KA, Erbelding EJ, Gaydos CA, Rompalo AM. Trichomonas vaginalis polymerase chain reaction compared with standard diagnostic and therapeutic protocols for detection and treatment of vaginal trichomoniasis. Clin Infect Dis. 2002;35(5):576-80.
16. Mahmoud A, Sherif NA, Abdella R, El-Genedy AR, El Kateb AY, Askalani AN. Prevalence of trichomonas vaginalis infection among Egyptian women using culture and latex agglutination: cross-sectional study. BMC Womens Health. 2015;15(1):7.

17. Garber GE. The laboratory diagnosis of trichomonas vaginalis. Canadian Journal of Infectious Diseases and Medical Microbiology. 2005;16(1):35-8.

18. Schwebke JR, Burgess D. Trichomoniasis. Clin Microbiol Rev. 2004;17(4):794-803.

19. Preethi V, Mandal J, Halder A, Parija SC. Trichomoniasis: an update. Tropical parasitology. 2011;1(2):73.

20. Service GS. 2010 population and housing census report: Ghana statistical service; 2014.

21. Shanti R, Potluri M. Sample size calculator: Raosoft Inc; 2009

22. Team RC. R: a language and environment for statistical computing; 2013.

23. Al-Saeed $\mathbf{W}$. Detection of trichomonas vaginalis by different methods in women from Dohok province, Iraq/Depistage de trichomonas vaginalis par differentes methodes chez des femmes de la province de Dahouk (Iraq). East Mediterr Health J. 2011;17(9):706-10.

24. Madhivanan P, Bartman MT, Pasutti L, Krupp K, Arun A, Reingold AL, et al. Prevalence of trichomonas vaginalis infection among young reproductive age women in India: implications for treatment and prevention. Sex Health. 2009;6(4):339-44.

25. Fernando SD, Herath S, Rodrigo C, Rajapakse L. Clinical features and sociodemographic factors affecting trichomonas vaginalis infection in women attending a central sexually transmitted diseases clinic in Sri Lanka. Indian journal of sexually transmitted diseases. 2012;33(1):25.

26. Verteramo R, Calzolari E, Degener AM, Masciangelo R, Patella A Trichomonas vaginalis infection: risk indicators among women attending for routine gynecologic examination. J Obstet Gynaecol Res. 2008;34(2):233-7.

27. Helms DJ, Mosure DJ, Metcalf CA, Douglas JM Jr, Malotte CK, Paul SM, et al. Risk factors for prevalent and incident trichomonas vaginalis among women attending three sexually transmitted disease clinics. Sex Transm Dis. 2008;35(5):484-8.

28. Sutton M, Sternberg M, Koumans EH, McQuillan G, Berman S, Markowitz L. The prevalence of trichomonas vaginalis infection among reproductive-age women in the United States, 2001-2004. Clin Infect Dis. 2007:45(10):1319-26.

29. Patil M, Nagamoti J, Metgud S. Diagnosis of trichomonas vaginalis from vaginal specimens by wet mount microscopy, in pouch TV culture system, and PCR. J Global Infect Dis. 2012;4:22.

30. Schwebke JR. Trichomonas vaginalis. Mandell, Douglas, and Bennett's principles and practice of infectious diseases: Elsevier; 2015. p. 3161-4. e1

31. Honigberg B. Trichomonads parasitic in humans: Springer Science \& Business Media; 2012.

32. Torian BE, Connelly RJ, Stephens RS, Stibbs HH. Specific and common antigens of trichomonas vaginalis detected by monoclonal antibodies. Infect Immun. 1984;43:270-5.

Ready to submit your research? Choose BMC and benefit from:

- fast, convenient online submission

- thorough peer review by experienced researchers in your field

- rapid publication on acceptance

- support for research data, including large and complex data types

- gold Open Access which fosters wider collaboration and increased citations

- maximum visibility for your research: over $100 \mathrm{M}$ website views per year

At $\mathrm{BMC}$, research is always in progress.

Learn more biomedcentral.com/submissions 